\title{
Hydrostaticity of Pressure Media in Diamond Anvil Cells *
}

\author{
YOU Shu-Jie(游淑洁), CHEN Liang-Chen(陈良辰), JIN Chang-Qing(靳常青)** \\ Institute of Physics, Chinese Academy of Sciences, Beijing 100190
}

\begin{abstract}
(Received 8 February 2009)
Hydrostaticity under high pressure of several materials from solid, fluid to gas, which are widely used as pressure media in nowadays high-pressure experiments, is investigated in diamond anvil cells. Judging from the $R$-line widths and $R_{2}-R_{1}$ peak separation of Ruby fluorescence, the inert argon gas is hydrostatic up to about 30 GPa. The behavior of silicon oil is found to be similar to argon at pressures less than 10 GPa, while the widening of $R$-lines and increase of $R_{2}-R_{1}$ peak separation at higher pressure loads indicate a significant degradation of hydrostaticity. Therefore silicon oil is considered as a good pressure medium at pressures less than 10 GPa but poor at higher pressures.
\end{abstract}

PACS: $62.50 .+p, 78.30 .-j, 81.40 . V_{W}$

Currently diamond anvil cell (DAC) has become one of the most powerful and popular tools in highpressure research since a pair of diamond anvils provide not only a convenient and simple way to maintain high pressure but also a good window for measurements. It has been successfully used in highpressure experiments like X-ray diffraction, optical spectroscopy, conductivity, magnetism and many other measurements. ${ }^{[1,2]}$ Pressure media play an important role in high-pressure measurements or experiments, especially in diamond anvil cell by greatly reducing the pressure gradient within the sample chamber and thus improving the uniformity of pressure action on the sample. The most important property of pressure media is, in turn, the hydrostaticity under high pressure, i.e. the ability to maintain a homogeneous hydrostatic pressure within the media since it governs the pressure distribution inside the sample chamber. In addition, it should be stable and inert or does not react with the sample during the measurement except for few special experiments, so that no signals affecting the result of the measurement will be emitted by the media, as well as easy to use from both cost and operation viewpoints. Unfortunately, for most pressure media, pressure gradient still appears even at very low pressures and becomes worse as pressure increases ${ }^{[3-5]}$ which makes the selection of appropriate pressure media for high-pressure experiments in DAC a severe task.

In this work we select three materials, i.e. sodium chloride $(\mathrm{NaCl})$, silicon oil and argon, for comparison, which are widely used in high-pressure research. Sodium chloride is solid in room temperature and atmospheric pressure that makes it very easy to load. It appears mainly in high-temperature high-pressure experiments like laser heating, where it serves not only as the pressure medium but also as the heat insulator. ${ }^{[6]}$ At room temperature it is sometimes used in the Raman spectroscopy measurement since it does not provide Raman active vibrations. Sili- con oil is a regular fluid pressure medium like the 4:1 methanol-ethanol mixture while it is less volatilizable and easier to conduct that makes it more favorable sometimes. We use silicon oil as medium instead of conventional methanol-ethanol mixtures in highpressure X-ray diffraction especially when the sample is sensitive to water and hydroxy compounds. It has been reported to have few weak peaks in Raman measurements ${ }^{[7]}$ and keep well hydrostatic up to $64 \mathrm{GPa}^{[8]}$ as well as argon, the commonly used inert gas in high-pressure research. Argon is a relatively cheap and easily liquefied inert gas with a reported small pressure difference of $1.7 \mathrm{GPa}$ at pressure of about $75 \mathrm{GPa} .{ }^{[9]}$

These three media are convenient and widely used in high-pressure research so that it is necessary to systematically study their performance under high pressure. In this work, we investigate capability of maintaining hydrostaticity under pressures up to about 30 $\mathrm{GPa}$ in these three media respectively by measuring the ruby fluorescence in each of them. Two criteria are employed: (1) the full width half maximum (FWHM) of $R$ line of ruby fluorescence; ${ }^{[10]}$ (2) the splitting between the ruby $R_{1}$ and $R_{2}$ fluorescence lines. ${ }^{[11]}$ Based on the results we discuss the suitability of each material as pressure medium under various conditions.

A symmetrical Mao-type diamond anvil cell is used to achieve high pressure in this work. The diameter of the diamond culets is about $300 \mu \mathrm{m}$. A piece of T301 stainless steel that serves as the gasket is preindented to a thickness of about $30 \mu \mathrm{m}$ from the initial $250 \mu \mathrm{m}$. A hole of $120 \mu \mathrm{m}$ in diameter is drilled in the middle of the pre-indented gasket. We simulate the real situation of high-pressure experiment by putting a chip of sample (50-70 $\mu \mathrm{m}$ in diameter) in the middle of the sample chamber. In the margin between the sample and the chamber, small ruby powder of approximately $15 \mu \mathrm{m}$ in grain size is loaded to monitor the pressure. ${ }^{[12]}$

*Supported by the National Key Basic Research Program of China under Grant Nos 2005CB724402 and 2007CB925003, and the National Natural Science Foundation of China under Grant No 10820101049.

**Email: JIN@aphy.iphy.ac.cn

(C) 2009 Chinese Physical Society and IOP Publishing Ltd 

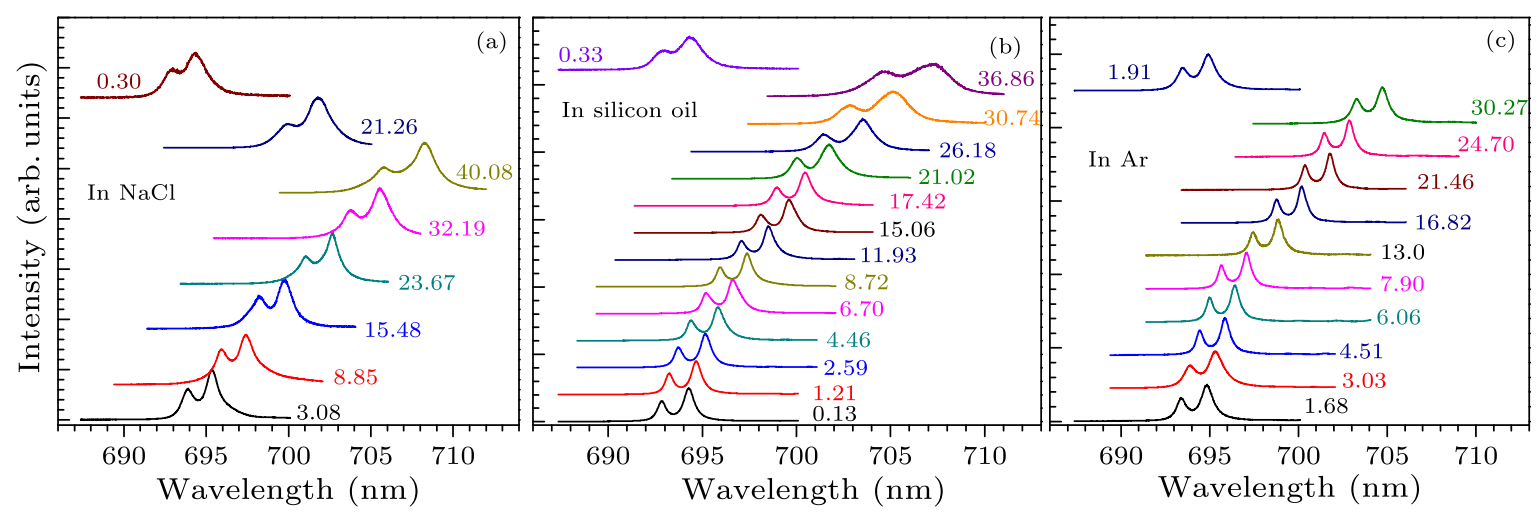

Fig. 1. The representative fluorescence spectra of ruby within each pressure medium: (a) in sodium chloride; (b) in silicon oil; (c) in argon. All the intensities are unified by the intensity of $R_{1}$ line. The colored number beside each spectra is the pressure computed from the $R_{1}$ line in the unit of GPa.

The detailed procedure of loading sample and the pressure medium depends on the pressure medium used in the experiment:

(1) For soft solid pressure medium such as sodium chloride $(\mathrm{NaCl})$, the medium is first dried and ground to fine powder then pressed into thin chips. One $\mathrm{NaCl}$ chip is loaded on the bottom of the sample chamber, sample and ruby chips lays over it. On the top of the chamber, another $\mathrm{NaCl}$ chip is loaded to form a NaClsample- $\mathrm{NaCl}$ sandwiched structure.

(2) For liquid pressure medium which is not highly volatilizable, for example silicon oil, the sample and ruby chips are placed in the sample chamber after it has been half filled with silicon oil, a very small quantity of the fluid is then added to hold the sample in the middle of the chamber.

(3) For gases that are easily liquefied like argon, a convenient apparatus for cooling and loading the cryogenic fluid has been designed and put into use. The diamond cell is assembled and cooled with the sample and ruby chips preloaded into the sample chamber. The cryogenic fluid is filled into the sample chamber by our handmade apparatus.

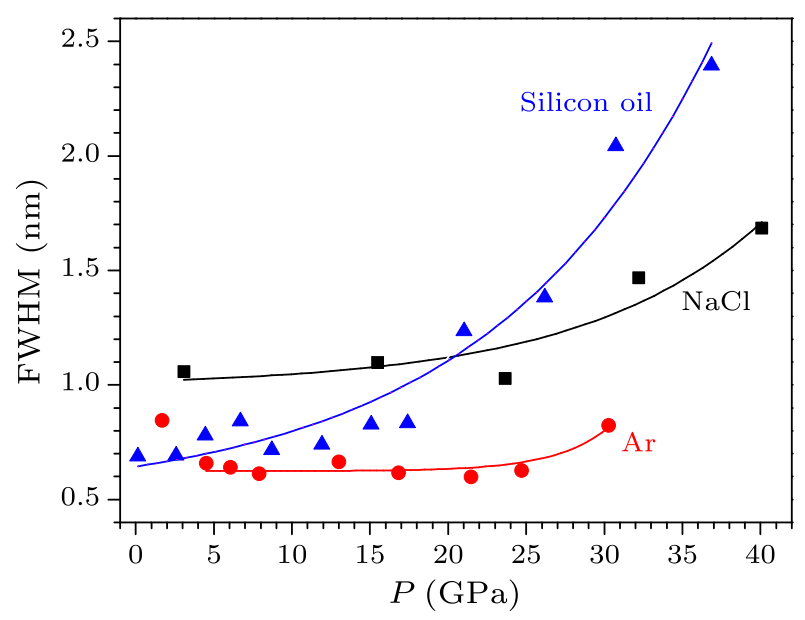

Fig. 2. FWHM of ruby $R_{1}$ line within each pressure medium the solid line here is only a guideline for visibility.

Ruby fluorescence was measured as soon as the pressure loaded with the relaxation discounted using an HR 800 Raman spectrometer with 1800 grooves $/ \mathrm{mm}$ holographic grating. The $532.8 \mathrm{~nm}$ (in wavelength) excitation laser is focused on the sample with a ZEISS $20^{*}$ objective of a $25 \mathrm{~mm}$ working distance. The time of scanning is $1 \mathrm{~s}$. Different grades of attenuation are used to obtain sufficient fluorescence intensity.

To ensure that the fluorescence data are obtained under the same condition and suitable for comparison in each run, we measure the fluorescence of the ruby chip that located about $50 \mu \mathrm{m}$ to the center of diamond culet in the case that ruby chips shift as pressure loads.

The fluorescence and calibration peaks were fitted using the program Origin. Voigt profile 1 was used to simultaneously fit both the ruby $R_{1}$ and $R_{2}$ peaks. The fits appear to be sensitive to the background and the range of simulation. Hence a linear background and symmetric data range were introduced in the procedure of peak fitting.

The fluorescence spectra of ruby within each pressure medium are plotted in Fig. 1. The intensity is normalized by the intensity of $R_{1}$. Figure 1 (a) gives the spectra in the pressure medium of $\mathrm{NaCl}$. A slight broadening of both $R_{1}$ and $R_{2}$ lines and a slow increasing of $R_{1}-R_{2}$ separation occur as pressure loads. The broadening of $R$ lines is much prominent at pressure above $12 \mathrm{GPa}$ in the case of silicon oil, as shown in Fig. 1(b). Apparently no significant change is observed in fluorescence of ruby in the medium of argon up to 30 GPa (Fig. 1(c)).

The pressure is calculated using the center of $R_{1}$ line (here noted as $\lambda$ ) by the equation of

$$
P(\mathrm{GPa})=1904 / B^{*}\left[(\lambda / 694.24)^{B}-1\right],
$$

for $\mathrm{NaCl}$, we choose $B=5$ (non-hydrostatic), for silicon oil and argon, we choose $B=7.665$ (quasihydrostatic), although little deviation of pressure was obtained for different $B$.

The FWHM of ruby $R_{1}$ line, reported to be sensitive to stresses, is plotted as a function of pressure 
in Fig. 2. No sign of broadening appears in argon up to $25 \mathrm{GPa}$, indicating that pressure distribution is well hydrostatic in the sample chamber. In silicon oil, the FWHM of $R_{1}$ line keeps steady at pressure $<15 \mathrm{GPa}$ but increases sharply when pressure gets higher. This is possibly caused by the solidification of silicon oil that induces an increase of both hardness and shear strength. In the case of sodium chloride, the initial FWHM is larger than that in silicon fluid and argon. Later it increases more slowly than that in silicon oil.

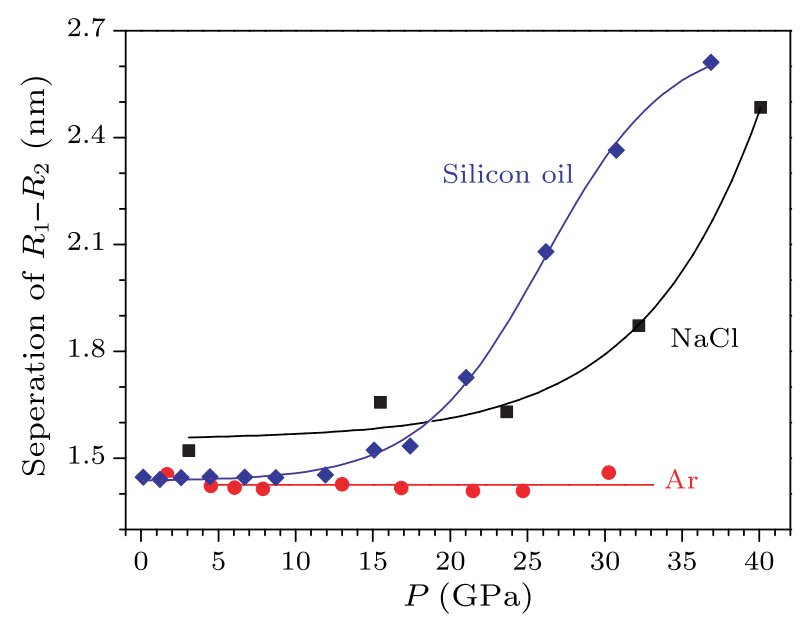

Fig. 3. Separation of ruby $R_{1}-R_{2}$ lines within each pressure medium.

In all the three pressure media, the FWHM of $R_{1}$ line increases and fluctuates in small ranges as pressure loads. This phenomenon is possibly due to the position shift of the ruby chips scanned. The splitting of $R_{1}-R_{2}$ peak of ruby fluorescence is also an indicator of hydrostaticity as reported. Therefore we also plot the separation of $R_{1}-R_{2}$ fluorescence lines in each medium as shown in Fig. 3. Behavior of the separation vs pressure is substantially similar to the FWHM except a steep cliff at pressure higher than $30 \mathrm{GPa}$ in $\mathrm{NaCl}$, implying a poor hydrostatic status in the sample chamber. It has been known for a long time that the structure of sodium chloride can trans- late from B1 to B2 (CsCl type) at about $29 \mathrm{GPa}$ with a remarkable increase of bulk modulus. This should be the explanation to the degradation of hydrostaticity.

In conclusion, we have investigated the fluorescence of ruby under high pressure in pressure media $\mathrm{NaCl}$, silicon oil and argon using a symmetrical Maotype diamond anvil cell. Utilizing of width of ruby $R$ line and separation of the $R_{1}-R_{2}$ peaks as the indicator, we figure out the status of hydrostaticity in these media as follows: Clearly argon is the best hydrostatic medium at pressure of about $30 \mathrm{GPa}$, though its applicability is sometimes restricted by the sophisticated loading procedure. Consistent with the result of Liu et al., ${ }^{[13]}$ silicon oil is also good hydrostatic at pressure smaller than $15 \mathrm{GPa}$. Sodium chloride is relatively poor regarding hydrostaticity while it is suitable for Raman measurement since it does not have the first order Raman vibration in both B1 and B2 phases.

\section{References}

[1] Jayaraman A 1983 Rev. Mod. Phys. 5565 Mao H K and Hemley R J 1994 Rev. Mod. Phys 66671

[2] Eremets M I 1996 High Pressure Experimental Methods, (Oxford: Oxford University Press)

[3] Meade C and Jeanloz R 1988 J. Geophys. Res. 93 3261-9

[4] Meade C and Jeanloz R 1990 Phys. Rev. B 42 2532-5

[5] Angel R J, Bujak M, Zhao J, Gatta G D and Jacobsen S D 2007 J. Appl. Cryst. 4026

[6] Hu J Z, Xu J, Somayazulu M, Guo Q, Hemley R and Mao H K 2002 J. Phys.: Condens. Matter 1410479

[7] Ragan D D, Clarke D R and Schiferl D 1996 Rev. Sci. Instrum. $\mathbf{6 7} 494$

[8] Shen Y R, Kumar R S, Pravica M and Nicol M F 2004 Rev. Sci.Instrum. $\mathbf{7 5} 4450$

[9] Mao H K, Bell P M, Shaner J W and Steinberg D J 1978 J. Appl. Phys. 49 3276-83

[10] Piermarini G J, Block S and Barnett J D 1973 J. Appl. Phys. 445377

[11] Asaumi K and Ruoff A L 1986 Phys. Rev. B 33 5633-6

[12] Mao H K, Xu J and Bell P M 1986 J. Geophys. Res. 91 4673-76

[13] Liu H Z, Hu J Z, Shu J F, Häusermann D and Mao H K 2004 Appl. Phys. Lett. 851973 